\title{
Automated Detection of Multiple Sclerosis Lesions in Normal Appearing White Matter from Brain MRI: A Survey
}

\author{
Manoj V. Khatokar ${ }^{1}$, M. Hemanth Kumar ${ }^{2}$, K. Chandrahas ${ }^{3}$, \\ M. D. Swetha ${ }^{4}$ and Preeti Satish ${ }^{5}$ \\ ${ }^{1,2 \& 3}$ UG Scholar, Department of Computer Science and Engineering, \\ ${ }^{4}$ Assistant Professor, ${ }^{5}$ Professor and Head, AI\&ML Department, \\ Dayananda Sagar College of Engineering, Bengaluru, Karnataka, India \\ E-mail: manojvkhatokar@gmail.com
}

\begin{abstract}
Multiple Sclerosis is an inoperable disease of the Central Nervous System (CNS) that irritates the myelin sheath by forming lesions. This affects all organs of the CNS; the vital of them is the brain. This disease can be detected by diagnosis like Magnetic Resonance Imaging (MRI). It is a non-invasive diagnostic test that provides detailed images of the soft tissues of the body. Out of the different variations of MRI, MS lesions are predominantly visible in the DTI (Diffusion Tensor Imaging) variant of MRI. DTI gives enhanced visualization of normal-appearing white matter tracts of the organs, hence providing a better image of the MS lesion. In this paper, the latest methodologies regarding the identification of the MS lesions in MRI scans like T2 FLAIR or DTI, using automated techniques like deep learning, computer vision, neural network and many more are surveyed. Furthermore, this paper consists of a proposed model which would focus on correlating the lesions found in DTI scan with the basic MRI scan like T2. It would identify the MS lesion in DTI scan and eventually highlight that lesion position in the T2 image scan. This would help radiologist in a way to effectively handle multiple MRI scans.

Keywords: Central Nervous System (CNS), Multiple Sclerosis (MS), Lesion, Magnetic Resonance Imaging (MRI), Diffusion Tensor Imaging (DTI), T2 Scan
\end{abstract}

\section{INTRODUCTION}

The nerves of the Central Nervous System (CNS) are composed of sheaths of myelin. They are sleeves of fatty tissue that protect the nerve cells, which are responsible for the message transportation in the CNS. This myelin sheath gets damaged in a situation when an immune-mediated inflammatory disease, called Multiple Sclerosis (MS), has occurred. MS is a chronic neurological disease of the CNS, which attacks the myelin sheath of the nerve cell. When these myelin sheaths are destroyed, tissue scars are formed known as sclerosis. This phenomenon, which tears down the myelin sheath, is popularly termed demyelination. These areas in the tissues form lesions and result in nonconductance of the impulse to and from the brain. It is commonly observed to afflict the young population whose age ranges from 25 to 45 . It is a challenge to diagnose and treat MS patients for its clinical expression is variable. The countries with the highest prevalence of MS include Canada, San Marino, Denmark, and Sweden. It is also prominently observed in regions of New Zealand, Australia, and the United States of America. The exact causes for this autoimmune disease have not yet been identified and is still a topic understudy in the fieldofNeurology. However, the symptoms are known to range from person to person. Some of the usual symptoms include fatigue, pain, bladder and bowel problems, sexual dysfunction, movement and coordination difficulties, vision and cognition changes, and emotional or mental health problems. Nonetheless, MS discretely affects eachindividual.Due to these complications and the unpredictable nature of MS, a highly powerful and non-invasive medical imaging technique has to be considered to diagnose this disease. Magnetic Resonance Imaging (MRI) is a method of diagnosis that uses strong magnetic fields and radio waves to produce detailed images of the inside of the human body. MRI scans help to visualize almost any part of the human body, including the most complex structured organ, the brain. MRI has proven to be the most promising method to identify MS. MRI provides a better soft- tissue contrast image of MS lesions and can differentiate better between fat, water, muscle, and other soft tissues. However, MS being an unlikely and unpredictable disease, is visible in several sequences of MRI. The regular MRI scans used to identify MS lesions are T1-weighted, T2-weighted, T2-FLAIR (Fluid Attenuated Inversion Recovery) weighted, PD (Protein Density) weighted, and Diffusion-weighted. Although all abovesequencesare used to detect MS lesions, some have their benefits. However, T2-weighted, T2-FLAIR, and Diffusion-weighted imaging protocols have increased levels of accuracy relatively. Among these protocols, the Diffusion-weighted technique outperforms in providing clear lesion images. Diffusion- weighted images are helpful to visualize vascular strokes in brain tissues. These images provide the structural damage of the tissue which cannot be portrayed by other conventional sequences such as T1weighted, T2-weighted. One of the diffusion-weighted techniques known as Diffusion Tensor Imaging (DTI) is considered as the 'gold standard' among all the MRI sequences for the identification of MS lesions.

DTI is a diffusion imaging technique that generates images based on the resulting data that uses the diffusion of water molecules to give contrast in the output images. DTI is applied when a particular tissue of the brain or any organ has its internal structure of fibres being analogous to the 
anisotropy of some crystals. It is primarily used for the study of white matter lesions and diseases regarding any tissue of the body. Molecular diffusion in tissues is not free but reflects interactions with many obstacles, such as macromolecules, fibres, and membranes. In regular circumstances, the free molecule of water has an equal movement in all directions in an isotropic manner. While their mobility in tissues is anisotropic due to the restriction of their movement towards the distinguished direction.

Therefore, this preferential motion depicts the path of the water molecules in tissues, which provides the enhanced detailing of the micro structural components in those tissues. Water motion in brain tissues is irregular due to several fibres. In segments like the white matter, movement is limited to a few extents, while in grey matter, this is furthermore controlled. In parts like the cerebrospinal fluid (CSF), there is plenty of room to man oeuvre for the water motion. MS causes various compulsive processes, forming lesions, and other changes in the usual brain structure.

This influences the regular water movement and provides a comprehensible view of the affected portion of the brain. This motion is captured by diffusion imaging techniques, in particular Diffusion Tensor Imaging (DTI). The diffusion tensor model is a rather simple model of the diffusion process, assuming homogeneity and linearity of the diffusion within each image voxel. From the diffusion tensor, diffusion anisotropy measures such as the Fractional Anisotropy (FA), can be computed. DTI information mainly holds the basis of water movement in the brain, where FA measurements are excluded. This gives the unidirectional diffusion measurement and thus, gives the prominent visual of the microstructure of the brain. Although this diffusion method was started in 1984, radiologists are experiencing difficulties in spotting the MS lesions inMRI.

Manual segmentation of MS lesions by experts is an ideal scenario in the detection of MS. However, there are complications in identification. Few complications include the following. An expert may mark out the lesions differently on the same MR scans provided to him at different times, due to human error, and there is a significant difference between the two segmentations performed by different experts. Next, considering the huge number of MR scans available for training a neural network, there are only a handful number of experts available for manual segmentation. Finally, experts need to spend a lot of timeconcentrating on the segmentation, which they can productively use for other important diagnosis tasks. Due to these reasons, an automated alternative of segmenting the lesions is the need of the hour, to assist the experts in making the task of identifying MS lesions effortless.

There are a lot of ways to arrive at an automated solution to the above-stated problem, while machine learning techniques such as supervised and unsupervised models are one among them. Some supervised models like K-nearest neighbours, artificial neural networks, random decision forests, and Bayesian framework, and unsupervised models like clustering aim to detect lesion voxels as outliers in major MRimages.

However, deep learning has proven to be the most effective when compared to the above models.One of the popular deep learning approaches includes convolution neural networks (CNN). Convolution neural networks (CNN) are being increasingly used in brain MRI image analysis for the segmentation of brain tumours, tissue, or pathological lesions. The architecture of $\mathrm{CNN}$ is analogous to the neurons and their connections to one another in the human brain. CNN's are mostly used in the identification of MS lesions due to their strong architecture and feature extraction power concerning images. CNN is designed to work with two-dimensional data, although it can be applied to one and three-dimensional data. The pre-processing of the data required for CNN is quite less when compared to other algorithms. Each image that is fed to the CNN is composed of pixels, the main role of the CNN is to reduce these pixels to a lower amount. The kernel is the main part of CNN architecture that plays a vital role in feature extraction and image reduction in terms of pixels to process the image more easily and without losing much of its features. The kernel takes strides from one pixel to the other based on the stride parameter set by the user, until the whole image is traversed. The objective of the kernel here is to extract the high-level features of the image like the edges, from the input image. While moving from one set of pixels to another, it applies pooling, which aggregates all the pixels present inside the kernel/filter into one pixel.

This reduces the spatial size of the convoluted feature by $\left(\mathrm{x}^{*} \mathrm{y}\right)-\left(\mathrm{x}^{\prime *} \mathrm{y}^{\prime}\right)$, where $\left(\mathrm{x}^{*} \mathrm{y}\right)$ is the original dimension and $\left(x^{\prime *} y^{\prime}\right)$ is the final dimension of the convoluted feature. The next step is to flatten the feature image into a column vector. This in turn is fed into a feed-forward neural network and back propagation is applied during the training process in every iteration. Throughout the epoch completion, the model can clearly distinguish between highlevel and low-level features and classify them using the soft-max classification technique.

There are innumerable approaches made to achieve the automatic diagnosis of the MS lesion from MRI sequences. These emphases on finding the lesions either in T2-FLAIR images or in diffusion images - DTI. Although these methods are unique and acceptable, they are confined to only one sequence. While they depict MS lesion in one sequence, that lesion can be an unusual foreign particle mass without MS metrics. Those methods fail to provide true identification of MS lesions. Henceforth, a comparison study between the basic sequence like T2-weighted and the 'gold standard' sequence - DTI, should be made. Thus, the optimal model would be to analyse both sequences (T2weighted and DTI). MS lesions from the DTI scan will be extracted by the CNN model, and eventually, it is highlighted at the same position in the T2-weighted image of the respective scan. This paper reviews the up-to-date 
methodologies used to extract MS lesions from major MRI sequences along with their importance and drawbacks. In addition, the difference between these techniques and the proposed model of this paper would be listed.

\section{SUMMARY OF PREVIOUS WORKS ON MS LESION DETECTION}

At various stages, the process of automated detection experienced dereliction. However, in the work done at [1], the datasets of different multi-channel brain MR scans including, T1-w, T2-w, PD-w, and FLAIR of 60 patients, where 36 of them were diagnosed with new T2-w lesions. The proposed model consists of a neural network with two main parts for the segmentation of the MS lesions. Initially, the U-net blocks, which are fast and precise convolution neural network architecture for segmentation of images, which outperforms the former best method, the sliding window convolution network. These U-net blocks learned the deformation fields (DFs) and non-linearly registered the baseline image to the follow-up image for each input modality. Secondly, these learning's from the DFs are taken into consideration along with the baseline and follow- up images, which were then fed to the second part of the neural network. The second part is also a U-Net model that is responsible for the final detection and the segmentation of the MS lesions from the new T2-w images. The loss function incorporated in this network is the summation of two-loss functions in particular. One is the unsupervised loss function that is responsible for controlling the registration part of the network. The other loss function is a supervised loss Cross-Entropy function that is responsible for the segmentation part of the network. This loss function also applies a penalty based on the differences between the segmentation and ground truth annotated lesions. Given the sensitivity and lower number of false positives in segmenting the lesions, the proposed model may be appropriately used for the detection of MS lesions.

As the technology advances, there are numerous automated procedures for MS lesion extraction from MRI. But, all these methods belong to either global extraction or patchbased extraction. Both methods have a few setbacks since they cannot identify tiny MS lesions in MRI. Hence, Yeeleng et al., proposed in [2] that using Synergy Net with the U-Net architecture embedded with RCNN (Regionbased Convolution Neural Network) bestow a radical change in the segmentation process. The SynergyNet comprises procedures linking networks of identification models for discrete lesion sizes. This model was trained on 765 longitudinal MRI scans of around 261 MS patients. T2weighted and 3D FLAIR images were included, while the result was depicted on the FLAIR scans alone. This framework has a $2.55 \%$ increase in Dice score, with a $20 \%$ decrease in false-positive metrics. Through the study in [2], it acknowledges that the usage of the fusion of segmentations of all sizes aggregates to a more robust application for the identification of MS lesions of any proportion. Since the model does neglect the usage of DTI scans, it provides excellence in the extraction of the lesion, while lacking to examine whetherthatlesion is MS or not. The proper definition of the MS lesion can be obtained when compared DTI segmented lesions are placed regarding other regular sequences and scrutinized. Our implemented work focuses on MS lesion recognition by DTI with the reference to T2-weighted scans and provides the results of T2-weighted scans. This may be handy for many radiologists as they would be not encouraged to analyse the sequences of the diagnosed patient.

Yue et al., in [3] propose the use of the Multiplicative Intrinsic Component Optimization (MICO) algorithm on multi-channel MRI scans for the identification of the MS lesions. For the various channels, distinct weights are provided, where more importance is given to T1-weighted and FLAIR images. This model consists of pre-processing where all unnecessary image pixels are filtered, followed by the model application through multi-channel MRI, and eventually, checking on the convergence criterion. Previously, MICO was applied to a single channeled MR image. But, in [3], this algorithm was extended to train on multichannel MRI scans, which provides a drastic difference in the results. Having a very small number of parameters (around16) provides robust overall performance. This method of MICO algorithm on multichannel was tested on the images from the MICCAI 2008 MS Lesion Segmentation Challenge. Although the results were better than the conventional method, it highlighted a few regions of false MS lesions. This model has restricted the input of T1-weighted and FLAIR images, which can be accounted for as a major drawback. As discussed above, instead of considering the 'gold standard' - DTI sequence, this model falls short to lay out the desired segmentation results. In our work, DTI will be considered along with other sequences of enriched outputs.

Amongst various emerging techniques of machine learning, deep learning has a vast utilization ranging from facial recognition systems to autonomous car development. The noteworthy application of this branch is the neural networks. In [4], Rafaat et al., have found that the fully convolutional neural network (FCNN) is obliged in segmenting the affected lesions of the MS victim. Their work helps to identify the T2-hyperintense MS lesions from the MR images. With around a thousand MRI scans, the model achieved an enriched performance of 95\% confidence limit. This was achieved due to the substantial multi-center dataset and the four-dimensional multimodal MR images. Diverse images helped to deliver a highly computational and more accurate model. When a large and heterogeneous dataset is applied to an FCNN model, the results have great precision. This study depicted that FCNN has an impressive impact on the segmentation of MS lesions. However, this technique too is applied to a particular MRI protocol. Furthermore, this study portrays the drawback of the non-availability of "ground truth" identification on these large datasets. For their huge dataset, the manual discerning of the MS lesions for each MRI scan 
is required. However, this turns out to be a very tedious task for a radiologist or a team of radiologists to identify the MS lesion boundaries in all the images of that enormous data. Besides, only the T1-weighted images were utilized in this study, and the cortical lesions were excluded from the dataset. This may deliver erroneously detected regions in the scan, due to neglecting the usage of DTI scans. In our implemented method, we would use a similar methodology, replacing the T1-weighted by DTI images.

The proposed work of [5] has a neural network that is based on the fact that the results should be classified into four clinical profiles. Beginning from the structural connectivity data, derived from the diffusion tensor imaging and shown as a graph, the evaluation of the performance of the classification is done using unweighted and weighted connectivity matrices. Further, the responsibility of the graph-based features is investigated for better classification and characterization of the MS lesions. The dataset consisted of 90 MS patients among which, 12 were clinically isolated, 30 were relapsing-remitting, 28 were secondary-progressive, and 20 were primary-progressive.

Additionally, 24 of the patients were taken as healthy controls for the study. The work of [5] not only shows the high performance in the classification of the pathology but also that local graph metrics do not improve the classification process, suggesting that the latent feature of the neural network plays a vital role in the classification process alone. It is also observed that the weights that show brain connections in the graph preserve information to discriminate between various clinical forms. Ref [6] Showed a novel approach towards detecting MS lesions in FLAIR sequences of MR scans. The objective is to develop an automated deep-learning approach for the segmentation of abnormalities on FLAIR, using a U-net convolutional neural network architecture using $3 \mathrm{D}$ volumes.

Deep convolutional encoder networks are the main idea behind achieving the task of segmentation in the work of [7]. The neural network of [7] is trained by applying both feature extraction and prediction in the same process. Doing so, makes the model automatically learn features from a collection of image types that in turn enhance the accuracy of the model. Instead of using a path-based approach for segmentation and training the model, here, the researchers have used the whole image for training the model. The reason behind this approach is that the training period can be drastically reduced and eliminates redundant calculations where patches overlap and need to select representative patches. Doing so allows researchers to scale up the model as the image resolution increases. The lesions are generally treated as an outlier class to model healthy tissue.

Coming from the network architecture, it comprises three layers, an input layer which contains image voxels of different modalities, a convolution layer that is responsible for creating a feature map, a de-convolution layer that uses the feature map to draw a lesion mask and thereby classify each voxel of an image in a single operation. A novel approach used in [7] is to not use the traditional approach of back propagation of the sum of squared differences of the predicted and expected outputs because if one class is extensively underrepresented, here lesions, which normally comprise less than one percent of the input image voxels, the model will train by neglecting the minority class completely. The novel idea here is to use an objective function that uses a weighted combination of sensitivity and specificity, structured to handle underrepresented classes. This formulates to allow stable gradient computations. The model is evaluated on the publicly labelled class from the MS lesion segmentation challenge from the 2008 dataset. The results were comparable to state-of-the-art methods.

The main hypothesis behind the study of [8] is that diffusion basis spectrum imaging (DBSI) can help in addressing MS lesion heterogeneity and that lesion-defining patterns can be discovered with the help of the DBSI metrics. The hypothesis is proven using a deep learning algorithm using neural networks and some of some imaging methods including diffusion-weighted imaging, magnetization transfer imaging, and standard conventional MRI sequences. A total of 499 regions of interest were labelled as persistent black holes (PBH), persistent grey holes (PGH), acute black holes (ABH), acute grey holes (AGH). The dataset collected in [8] had a total of 43,261 imaging voxels from 38 MS patients. As usual, a generic approach of splitting the dataset into training, testing, and validation was done. However, the ratio in which they were split was in $8: 1: 1$, respectively. The imaging voxels were included in the training and validation datasets.

Whereas, imaging voxels were separated from the test datasets. The model of [8] was developed using the Tensor Flow framework in python. The diffusion metrics that were calculated with the neural network were fiber fraction, fiber fractional anisotropy (FA), fiber $\mathrm{AD}$, fiber $\mathrm{RD}$, restricted isotropic diffusion fraction (restricted fraction), hindered isotropic diffusion fraction (hindered fraction), free isotropic diffusion fraction (water fraction) and normalized b0 intensity. DTI metrics include ADC, FA, AD, and RD. The model of [8] is a fully connected neural network, having the most popular softmax function as the final layer to create a total of 5 different classes to distinguish the input images from. After a lot of manual tuning of the hyper parameters and grid search techniques, an optimal network architecture was obtained. The DBSI-DNN discriminated PBH, PGH, AGH, NBH, and NAWM with positive prediction rates of $91.3 \%, 83.4 \%, 90.1 \%, 92.3 \%$, and $97.9 \%$, respectively, and prediction accuracy of $93.4 \%$ in identifying the MS lesions.

CNN's perform very well in conditions where training data is abundantly available for positive samples. If the training data with positive samples is very less when compared to the negative samples, the chances of overfitting are very high, which is not acceptable in making crucial medical predictions. Since the number of labelled data available for 
training is less in the case of MS lesion segmentation, the study of [9] satisfactorily samples the training data and splits the training procedure into two different CNN network architecture, by constructing a pipeline with fewer parameters, while at the same time not giving up the accuracy of the model. The work of [9] shows that applying this kind of data augmentation effectively increases the segmentation accuracy of the proposed model. The obtained results are encouraging, yielding the CNN architecture closer to human-expert inter-rate variability.

The datasets of [10] included two different kinds, one, the publicly available ISBI 2015 Longitudinal MS Lesion Segmentation Challenge dataset, second, the private dataset obtained from the neuroimaging research unit (NRU) in Milan. The proposed network architecture consists of a twodimensional end-to-end convolution neural network (ResNet). The main idea behind the ResNet Model of [10] is to identify shortcut connections that prevent gradient vanishing and reduce computational complexity. The readily available ResNet50 was modified to work as a pixellevel segmentation network by adding a dense convolutional layer at the end of the network that does pixel-level prediction. The proposed CNN also includes a downsampling feature in the pipeline that is branched in multiple ways. This allows the network to encode information from multiple modalities separately.

The feature maps are combined from different stages of the network from different modalities with the help of multiscale feature fusion blocks. The pipeline is followed by multi-scale feature up sampling blocks to leverage information by upsizing the combined feature maps. On the private dataset, the proposed model made in [10] shows high performance and improvements in the MS lesion segmentation compared to other readily available public segmentation tools.

In the analysis of [11], MR intensity and white matter priors are used to extract candidate lesion voxels. Following which, a convolutional neural network is used for the task of final segmentation and false-positive reduction in [11]. The CNN used longitudinal data, which is a novel contribution in the field of MS lesion analysis. The study of [11] was done in mainly three steps, firstly pre-processing, where the acquired images, which are not registered and which have a different level of splicing and thickness, are converted to a commonly readable format and normalized in terms of intensity as well. Brain extraction from the images is also taken care of in the pre-processing stage and bias field correction is also applied. Secondly, candidate extraction is done as an initial filter to the lesion detection task.

In this, a small subset of lesion voxels is considered as an input to the next stage, the CNN model. Thirdly, CNN prediction, where the extracted lesions are fed as input to the CNN architecture, and the output of this phase is the lesion probability value per image voxel. Looking at the results obtained from [11], we can say that CNN that uses the longitudinal image data for training and prediction can produce better segmentation results than other CNNs. Several experimentations have stated the existence of cortical lesions in MS infected individual's acts as a dominant biomarker for MS disease. These lesions occur at the premature stages of this disease. Their existence provides a piece of prominent information to the radiologists regarding the development of MS lesions in the brain.

However, the identification of these lesion bodies in MRI scans through naked inspection by doctors has always been a hectic work. Hence, in [12], Francesco et al., put forth a machine learning model for the recognition of these cortical lesions along with the white matter lesions in two sequences of 3T MR images. These two sequences are, namely, the T2-weighted FLAIR sequence and the T1-weighted M2PRAGE (Magnetization Prepared Rapid Acquisition Gradient Echo) sequence. With the strength of 90 patients, an aggregate of 728 grey matter lesions and 3856 white matter lesions were piled up for the dataset. This work depicted that the conventional method of usage of a fully convolutional neural network (F- CNN) with a 3D U-Net framework failed to point out the smaller regions of cortical lesions in the brain MRI scans. Using approach used in [12], a decent score in the identification rate of $76 \%$ and a false positive of $29 \%$ was encountered.

This procedure aids in finding both the cortical and white matter MS lesions, which is obliging for the doctors. However, the model in [12] had a deficient in analysis, when both cortical and white matter lesions were to be highlighted, where they were in discrete sizes. Our proposed model would implement the identification of cortical lesions along with the already existing goal, as it might be of great use to the medical analysis. Throughout many processes, researchers have identified the MS lesions from MRI scans from a particular sequence, which is captured at a particular time. One time-stamped scans were used for the detection of lesions for this disease. Nils et al., further extended this segmentation process by the inclusion of two scans made at two separate time positions. Their work in [13] has elucidated that MR images from two different time schedules had a great impact on the exploration of this MS extraction method. The activity-based inspection and deep learning techniques made the process of MS diagnosis to become a step more manageable and simpler. Instead of a single framework, the dual-channeled working mechanisms of the CNNs from the points of two distinguished time frames are operated.

Though the performance of this model matches with the other CNN with U- Net architecture model, it excels over the run-of-the-mill methods. [13] Gives conceivable attention maps to the end-users which outperforms in various aspects. Nonetheless, the routine work for this study is strenuous. The data collected at a timestamp should be kept aside after the first phase of analysis. Subsequently, the diagnosis of the same patient has to be collected. This 
accounts for a laborious task for the medical staff. Consequently, this approach of two-path CNNs performed on MRI scans of a particular interval is not the ideal solution for this research. With numerous neoteric deep learning models, Ozdemir et al., pointed out the conventional method of detection of MS lesions. In [14], the supervised learning of the minimum Euclidean distancebased algorithm had a step ahead in performance criteria. This accounts for a light-weight automated model for the detection of lesions in MR images. The work in [14] was relayed mainly using three 2D-MRI protocols, T1-weighted, T2- weighted, and FLAIR modalities.

As the model was developed using the present-day techniques, this work proclaimed approximately 90\% specificity and accuracy. Around $65 \%$ sensitivity was achieved with this approach. This algorithm may propose superiority over deep-learning methods. Nonetheless, this model does not encourage the extraction of the MS lesion in the scans. Moreover, this participates only under the classification criteria, instead of the segmentation class. This model falls short of the proposed objective of this paper. Hence, we would implement the basic model of classification, but apply other advanced deep learning methods for the identification process. In [15], it has been proposed that a patch-wise RCNN must be for the segmentation of MS lesions. Pre-processing is done by removing the noise and other unnecessary artifacts that might hinder the identification of lesions. Later, the skull is stripped from the brain to remove unnecessary image data and bias field corrections are also applied appropriately.

Finally, the 3D patches of the lesions are extracted from the brain images and fed into the RCNN and trained accordingly. To improve the segmentation results, a neurofuzzy inference system (ANFIS) implemented to fuse the probability maps from different modalities.The methodology of CNN in the segmentation province of medical image processing has a considerable impact. Various proposed models have excelled in this aspect. Nevertheless, they have failed to provide better results across numerous MRI scans. Though their accuracy and precision are quite outstanding in one domain of MRI protocols, they are deficient while tested on images with more vibrant characteristics. Hence, Sergi et al., have come up with work in [16] where the intensity domain adaptation administration was performed. Their operation was executed on two public datasets of MS patients, one being the ISBI2015 challenge dataset while the other was the clinical dataset. Supervised-learning on the domain images was surveyed in this work of [16].

The effectiveness of this domain adaptation model in transferring previously acquired knowledge to later MRI scanned domains has a breakthrough. To summarize this work, one-shot domain adaptive was in mere comparison with manual handling of these scans. We might implement this methodology for the segmentation of MS lesions in DTI, while this can be enhanced by the usage of numerous other deep learning mechanisms.

\section{CONCLUSION}

Although region of interest (ROI) analysis was adopted in early MS studies, these methods discussed above proved to have some drawbacks; that is, it is time-consuming, operator dependent, and subject to partial volume artifacts; furthermore, it does not provide a global assessment of tissue damage. A large dataset is required in the positive training class to prevent the model to over fit. Since, usually, there is a lesser number of positive training samples, one of the many approaches taken is data augmentation. It is also observed that a pre-processing stage has to be implemented to get prediction accuracy and achieve faster learning rates. Though in this survey, discrete methods of computerized methods were proposed with an accuracy statement of greater weight age, they all fell flat in the correlation between the main lesion standards-DTI and FLAIR. Hence, we summon our project to form a bridge between the crucial modalities of the MR images. The model would pick up the MS lesions from the DTI scans, highlight the respective voxels in the 3D images of $\mathrm{T} 2$ or other preliminary sequences. This would enhance the work rate of the radiologists and would provide adequate information regarding the patients. Also, this would make the MS infected receive information regarding this disorder in the early stages.

\section{REFERENCES}

[1] Salem, Mostafa, Sergi Valverde, Mariano Cabezas, Deborah Pareto, Arnau Oliver, Joaquim Salvi, ÀlexRovira and Xavier Lladó, “A fully convolutional neural network for new T2-w lesion detection in multiple sclerosis," Neuro Image: Clinical, Vol. 25, No. 102-149, 2020.

[2] S. Vang, Yeeleng, Yingxin Cao, Peter D. Chang, Daniel S. Chow, Alexander U. Brandt, Friedemann Paul, Michael Scheel and Xiaohui Xie, "Synergy Net: A Fusion Framework for Multiple Sclerosis Brain MRI Segmentation with Local Refinement," In 2020 IEEE 17th International Symposium on Biomedical Imaging (ISBI), IEEE, pp. 131-135, 2020.

[3] Zhao, Yue, Shuxu Guo, Min Luo, Yu Liu, Michel Bilello and Chunming $\mathrm{Li}$, "An energy minimization method for MS lesion segmentation from T1-w and FLAIR images," Magnetic resonance imaging, Vol. 39, pp. 1-6, 2017.

[4] E. Gabr, Refaat, Ivan Coronado, Melvin Robinson, Sheeba J. Sujit, Sushmita Datta, Xiaojun Sun, William J. Allen, Fred D. Lublin, Jerry S. Wolinsky and Ponnada A. Narayana, "Brain and lesion segmentation in multiple sclerosis using fully convolutional neural networks: A large-scale study,” Multiple Sclerosis Journal, Vol. 26, No. 10, pp. 1217-1226, 2020.

[5] Marzullo, Aldo, Gabriel Kocevar, Claudio Stamile, Françoise Durand-Dubief, Giorgio Terracina, Francesco Calimeri and Dominique Sappey-Marinier, "Classification of multiple sclerosis clinical profiles via graph convolutional neural networks," Frontiers in neuroscience, Vol. 13, No. 594, 2019.

[6] Duong, Michael Tran, Jeffrey D. Rudie, Jiancong Wang, Long Xie, Suyash Mohan, James C. Gee, and Andreas M. Rauschecker, "Convolutional neural network for automated FLAIR lesion segmentation on clinical brain MR imaging," American Journal of Neuroradiology, Vol. 40, No. 8, pp. 1282- 1290, 2019.

[7] Brosch, Tom, Youngjin Yoo, Lisa YW Tang, David KB Li, Anthony Traboulsee and Roger Tam, "Deep convolutional encoder networks for multiple sclerosis lesion segmentation," In International Conference on Medical Image Computing and Computer-Assisted Intervention, pp. 3-11.Springer, Cham,2015.

[8] Ye, Zezhong, Ajit George, Anthony T. Wu, Xuan Niu, Joshua Lin, Gautam Adusumilli, Robert T. Naismith, Anne H. Cross, PengSun, and Sheng Kwei Song, "Deeplearning with diffusion basis spectrum 
imaging for classification of multiple sclerosis lesions," Annals of Clinical and Translational Neurology, Vol. 7, No. 5, pp. 695-706, 2020.

[9] Valverde, Sergi, Mariano Cabezas, Eloy Roura, Sandra GonzálezVillà, Deborah Pareto, Joan C. Vilanova, Lluís Ramió- Torrentà, Àlex Rovira, Arnau Oliver and Xavier Lladó, "Improving automated multiple sclerosis lesion segmentation with a cascaded 3D convolutional neural network approach,” Neuro Image, Vol.155,pp. 159-168, 2017.

[10] Aslani, Shahab, Michael Dayan, Loredana Storelli, Massimo Filippi, Vittorio Murino, Maria A. Rocca and Diego Sona, "Multi-branch convolutional neural network for multiple sclerosis lesion segmentation," NeuroImage, Vol. 196, pp. 1-15, 2019.

[11] Birenbaum, Ariel and Hayit Greenspan, "Multi-view longitudinal CNN for multiple sclerosis lesion segmentation," Engineering Applications of Artificial Intelligence, Vol. 65, pp. 111-118, 2017.

[12] La Rosa, Francesco, Ahmed Abdulkadir, MárioJoão Fartaria, Reza Rahmanzadeh, Po-Jui Lu, Riccardo Galbusera, Muhamed Barakovic, Jean-Philippe Thiran, Cristina Granziera, and Merixtell Bach Cuadra, "Multiple sclerosis cortical and WM lesion segmentation at 3T MRI: a deep learning method based on FLAIR and MP2RAGE," NeuroImage: Clinical, Vol. 27, No. 102335, 2020.
[13] Gessert, Nils, Julia Krüger, Roland Opfer, Ann-Christin Ostwaldt, Praveena Manogaran, Hagen H. Kitzler, Sven Schippling, and Alexander Schlaefer, "Multiple sclerosis lesion activity segmentation with attention-guided two-path CNNs," Computerized Medical Imaging and Graphics, Vol. 84, No. 101772, 2020.

[14] Cetin, Ozdemir, Volkan Seymen, and Unal Sakoglu, "Multiple sclerosis lesion detection in multimodal MRI using simple clusteringbased segmentation and classification, "Informatics in Medicine Unlocked, Vol. 20, No. 100409, 2020.

[15] Essa, Ehab, Doaa Aldesouky, Sherif E. Hussein and M. Z. Rashad, "Neuro-fuzzy patch-wise R-CNN for multiple sclerosis segmentation," Medical \& Biological Engineering \& Computing, Vol. 58, No. 9, pp. 2161-2175, 2020.

[16] Valverde, Sergi, Mostafa Salem, Mariano Cabezas, Deborah Pareto, Joan C. Vilanova, Lluís Ramió-Torrentà, ÀlexRovira, Joaquim Salvi, Arnau Oliver and Xavier Lladó, "One-shot domain adaptation in multiple sclerosis lesion segmentation using convolutional neural networks," NeuroImage: Clinical, Vol. 21, No. 10, 2019. 